\section{Caudate volume in offspring of patients} with schizophrenia

\author{
RAJAPR ABHAK ARAN RAJARETHINAM, AMEET UPADHYAYA, PON TSOU, \\ MARGIE UPADHYAYA and MATCHERI S. KESHAVAN
}

\begin{abstract}
Summary Caudate nuclei are smaller in drug-naive people with schizophrenia but larger in antipsychotic-treated patients. In this magnetic resonance imaging study we found volume reduction of right and left caudate by 8.9 and $8.1 \%$ respectively in 50 offspring without psychosis of patients with schizophrenia compared with 53 age- and gendermatched controls, providing new evidence that caudate volume reduction may be a trait-related abnormality in schizophrenia.
\end{abstract}

Declaration of interest None.

The caudate nucleus is a major target area for the subcortical dopamine projection system, which is implicated in the pathophysiology of schizophrenia. Caudate and other basal ganglia nuclei help regulate and organise the information flow between frontal lobes and the rest of the brain and play a major part in higher cognitive functions and movement (Middleton \& Strick, 1994). Disruptions in this system or lesions of the basal ganglia result in movement disorders and behavioural problems similar to schizophrenia (Heckers, 1997).

Previous studies have shown enlarged caudate in patients treated with dopamineblocking antipsychotics (Jernigan et al, 1991); however, a reduction has been reported among drug-naive patients (e.g. Keshavan et al, 1998) but it is not known whether this reduction precedes the illness. At-risk studies have shown brain abnormalities and behavioural deviances supporting neurodevelopmental pathology prior to psychosis (Lawrie et al, 2001; Rajarethinam et al, 2004; Job et al, 2005; Keshavan et al, 2005). We predicted that individuals at risk would have a smaller caudate nucleus than those with no family history of mental illness.

\section{METHOD}

Fifty young people (22 males and 28 females, mean age 15.4 years, s.d. $=3.6$ ) with at least one parent with schizophrenia and 53 healthy comparison participants $(27$ males and 28 females, mean age 16.5 years, s.d.=4.4) with no family history of mental illness participated from an ongoing study at the University of Pittsburgh. Other findings from this study have been reported elsewhere (Rajarethinam et al, 2004; Keshavan et al, 2005). The parental diagnosis in the high-risk group was ascertained with the Structured Clinical Interview for DSM-IV (SCID; Spitzer et al, 1992) and consensus clinical diagnosis. Comparison participants were similar in age, gender and socio-economic and geographical background but with no family history of mental illness. In the high-risk group 26 (57\%) had Axis I psychopathology and IQ was lower (control group, mean IQ 115, s.d.=12; high-risk group, mean IQ 103, s.d.=13; $F=7.184, P<0.011)$. None of the participants had psychosis. Seven participants were being treated with stimulants and four with antidepressants, but none with antipsychotics. The University of Pittsburgh institutional review board approved the study. All participants provided written consent; those under 18 years provided informed assent also. Participants aged 15 years or younger were evaluated with the Schedule for Affective Disorders and Schizophrenia for School-Age Children (K-SADS-PL version; Kaufman et al, 1999) and those over 15 were evaluated using the SCID. Anyone with a lifetime history of psychosis, significant neurological or medical illness, current substance use disorder, or any contraindication for magnetic resonance imaging (MRI) was excluded from participating in either group.

For MRI, $124 T_{1}$-weighted $1.5 \mathrm{~mm}$ coronal slices, without interslice gap, were obtained using a 1.5 T GE scanner (General Electric, Milwaukee, Wisconsin, USA) with three-dimensional spoiled gradient recall acquisition, matrix $256 \times 256 \times 192$, field of view $24 \mathrm{~cm}$, repetition time $25 \mathrm{~ms}$, and time to echo $5 \mathrm{~ms}$. Scans were reviewed to exclude structural abnormalities. The total brain volume was measured by a semi-automatic method using BRAINS2 software (Magnotta et al, 2002) and manually edited for accuracy. The interrater reliability between the four raters ranged from 0.98 to 0.99 .

Caudate volumes were measured using the artificial neural network application, a semi-automated tracing method in BRAINS2, with manual editing for accuracy by two trained raters (P.T. and M.U.). Measurement included the head and body of the caudate but not the tail. The test-retest reliability for each rater was established using a set of three scans: (P.T.) right caudate $r=0.98$, left caudate $r=0.99$; (M.U.) right caudate $r=0.99$, left caudate $r=0.95$. The interrater reliability (intraclass $r=0.94$ for both right and left caudate) was established using a set of nine scans. Differences in age and intracranial volume were examined by two-tailed unpaired $t$-tests. Multivariate analysis based on the general linear model was conducted with group status (at-risk $v$. control) and intracranial volume as predictor variables and caudate volumes as dependent variables.

\section{RESULTS}

Age and gender distribution were not significantly different between the groups. The mean total brain volume of the highrisk group $\left(1311.25 \mathrm{~cm}^{3}\right.$, s.d.=130.7) was significantly smaller than that of the comparison group $\left(1391.21 \mathrm{~cm}^{3}\right.$, s.d.=136.3, $F=9.223, \quad P>0.003)$. Analysis of covariance using brain volume as a covariate revealed that the right caudate was significantly smaller in the high-risk group $(F=4.014, P<0.05)$ and the left caudate showed a trend for reduction $(F=2.92$, $P=0.091$ ) (Fig. 1). The right and left caudate nuclei were smaller in the high-risk group by 8.9 and $8.1 \%$ respectively (right caudate: control mean volume $3.58 \mathrm{~cm}^{3}$, s.d. $=0.51$; high-risk mean volume $3.26 \mathrm{~cm}^{3}$, s.d. $=0.45$; left caudate: control mean volume $3.57 \mathrm{~cm}^{3}$, s.d. $=0.51$, high-risk mean volume $3.28 \mathrm{~cm}^{3}$, s.d. $=0.49$ ). Psychopathology or medication status did not have any significant effect on caudate volumes.

\section{DISCUSSION}

Our findings indicate that abnormalities in the caudate nucleus may be seen in young relatives of patients with schizophrenia. Individuals at risk for schizophrenia exhibit 
behavioural problems and brain abnormalities, suggesting that some form of the pathological process may begin before the onset of symptoms (Keshavan et al, 2005). However, not all of these at-risk individuals would develop schizophrenia; therefore, the observed neuroanatomical alterations may reflect a measure of familial risk or susceptibility. The conversion to psychosis may result from an interaction between such susceptibility factors and unknown environmental influences or developmental/maturational changes that may involve this system.

Our observations are consistent with studies of basal ganglia function in individuals at risk for schizophrenia. Adult firstdegree relatives of schizophrenia patients made more errors on an antisaccade task than a comparison group, suggesting a dysfunction of dorsolateral prefrontal cortex, caudate nucleus, or both (Clementz et al, 1994). Similarly, functional MRI studies have shown decreased activation of the caudate with antisaccade tasks in unaffected relatives (Raemaekers et al, 2006). These findings suggest that an alteration of the structural and functional integrity of corticostriatal neural networks may represent familial or premorbid risk of schizophrenia. It is conceivable that this network may have a role in other neurocognitive deficits such as attentional impairments found in at-risk individuals (Keshavan et al, 2005). In addition, in the context of conflicting research regarding the increase and decrease in caudate volume in relation to treatment or drugnaive status, our data clearly support the hypothesis that volume reduction rather than enlargement of the caudate nucleus is associated with the pathophysiology of schizophrenia. In contrast, Lawrie et al (2001) reported no difference in the caudate volumes in at-risk relatives (not

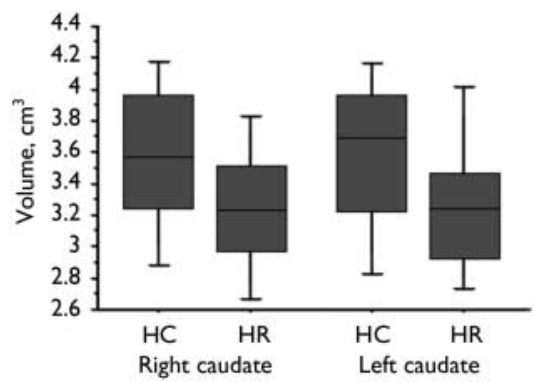

Fig. I Covarying for the total intracranial volume, the right caudate volume was significantly smaller in the high-risk group (HR) than in the healthy control group $(\mathrm{HC})(F=4.014, P<0.05)$, and the left caudate showed a trend for reduction $(F=2.92, P=0.091)$.

RAJAPRABHAKARAN RAJARETHINAM, MD, AMEET UPADHYAYA, MD, PON TSOU, BS, MARGIE UPADHYAYA, MD, Department of Psychiatry, Wayne State University School of Medicine, Detroit, Michigan; MATCHERI S. KESHAVAN, MD, Departments of Psychiatry, Wayne State University School of Medicine, and University of Pittsburgh School of Medicine, Pittsburgh, Pennsylvania, USA

Correspondence: Dr M. S. Keshavan, Department of Psychiatry, Wayne State University School of Medicine, 420I St Antoine Street UHC-9B, Detroit, Michigan 4820I,USA. Email: mkeshava@med.wayne.edu

(First received 27 July 2006, final revision 27 February 2007, accepted 19 March 2007)

offspring), some of whom were symptomatic.

To our knowledge, few studies have examined basal ganglia in asymptomatic, untreated, adolescent offspring who are at genetic risk of schizophrenia. High-risk studies enable investigation of neuropathology without the confounds of state-related illness manifestations and medication effects. The neuroanatomical specificity of the observed findings is unclear, but caudate volume reductions might be part of an abnormal corticostriatal network; we and others have found prefrontal and temporal cortical volume deficits in this population (Rajarethinam et al, 2004; Job et al, 2005). The precise mechanisms underlying caudate volume reduction are unclear, and may involve either a failure of normal development or an excessive pruning (Keshavan et al, 2005).

These findings are intriguing, but must be considered preliminary, need replication and may not be generalisable to non-familial forms of schizophrenia. Although the difference was modest, type I errors are unlikely as the sample was large. Our findings support the notion that smaller caudate is a marker of genetic susceptibility, but it is not known whether this abnormality is present at birth or becomes evident during childhood and adolescence. Prospective studies in high-risk individuals suggest that about $10-15 \%$ develop schizophrenia, although up to $40 \%$ develop schizophreniaspectrum psychopathology (ErlenmeyerKimling et al, 1997). Follow-up of these individuals will help elucidate the role of the caudate in premorbid vulnerability to and later progression into schizophrenia.

\section{ACKNOWLEDGEMENTS}

Support from National Institute of Mental Health grants $\mathrm{MH}$ 64023, 01180 (M.S.K.), NARSAD independent Investigator award (M.S.K.) and GCRC grant MOI RR00056. We thank Diana Dworakowski, MS, and Debra Montrose, PhD, for help with recruitment and assessment, and Jeffrey Nutche, BS, for image processing.

\section{REFERENCES}

Clementz, B. A., McDowell, J. E. \& Zisook, S. (1994) Saccadic system functioning among schizophrenia patients and their first-degree biological relatives. Journal of Abnormal Psychology, 103, 277-287.

Erlenmeyer-Kimling, L., Adamo, U. H., Rock, D., et al (1997) The New York High-Risk Project. Prevalence and comorbidity of axis I disorders in offspring of schizophrenic parents at 25-year follow-up. Archives of General Psychiatry, 54, 1096-1102.

Heckers, S. (1997) Neuropathology of schizophrenia: cortex, thalamus, basal ganglia, and neurotransmitterspecific projection systems. Schizophrenia Bulletin, 23 403-42I.

Jernigan, T. L., Zisook, S., Heaton, R. K., et al (199I) Magnetic resonance imaging abnormalities in lenticular nuclei and cerebral cortex in schizophrenia. Archives of General Psychiatry, 48, 88I-890.

Job, D. E., Whalley, H. C., Johnstone, E. C., et al, (2005) Grey matter changes over time in high risk subjects developing schizophrenia. Neurolmage, 25, 1023-1030.

Kaufman, J., Birmaker, B., Brent, D., et al (1999) Schedule for Affective Disorders and Schizophrenia for School-Age Children - Present and Lifetime Version (K-SADS-PL): initial reliability and validity data. Journal of the American Academy of Child and Adolescent Psychiatry, 38, 1065-1069.

Keshavan, M. S., Rosenberg, D., Sweeney, J. A., et al (1998) Decreased caudate volume in neuroleptic-naive psychotic patients. American Journal of Psychiatry, I55, 774-778.

Keshavan, M. S., Diwadkar, V. A., Montrose, D. M., et al (2005) Premorbid indicators and risk for schizophrenia: a selective review and update. Schizophrenia Research, 79, 45-57.

Lawrie, S. M., Whalley, H. C., Abukmeil, S. S., et al (200I) Brain structure, genetic liability, and psychotic symptoms in subjects at high risk of developing schizophrenia. Biological Psychiatry, 49, 8II-823.

Magnotta, V. A., Harris, G., Andreasen, N. C., et al (2002) Structural MR image processing using the BRAINS2 toolbox. Computerized Medical Imaging and Graphics, 26, 251-264.

Middleton, F. A. \& Strick, P. L. (1994) Anatomical evidence for cerebellar and basal ganglia involvement in higher cognitive function. Science, 266, 458-46I.

Raemaekers, M., Ramsey, N. F., Vink, M., et al (2006) Brain activation during antisaccades in unaffected relatives of schizophrenic patients. Biological Psychiatry, 59, 530-535.

Rajarethinam, R., Sahni, S., Rosenberg, D. R., et a (2004) Reduced superior temporal gyrus volume in young offspring of patients with schizophrenia. American Journal of Psychiatry, |61, ||2|-||24.

Spitzer, R. L., Williams, J. B., Gibbon, M., et al (1992) The Structured Clinical Interview for DSM-III-R (SCID). I: History, rationale, and description. Archives of General Psychiatry, 49, 624-629. 